Porras Soriano, Álvaro.

Estudiante Programa Doctorado Arte, Humanidades y Educación. Investigador perteneciente al Grupo: Interfaces Culturales. Arte de los Nuevos Medios. Departamento de Arte, Universidad de Castilla-La Mancha.

López Cleries, Gloria.

Personal Contratado Pre-Doctoral FPU2015, perteneciente al Grupo de Investigación: Interfaces

Culturales. Arte de los Nuevos Medios. Departamento de Arte, Universidad de Castilla-La Mancha.

\title{
Contradicciones del arte post-digital. Neomaterialismos vs. Infinitud.
}

\section{Disproofs of post-digital art. Neomaterialisms vs. infiniteness.}

TIPO DE TRABAJO:

Comunicación.

PALABRAS CLAVE:

Postinternet, post-digital, neomaterialidad, arte contemporáneo, arte conceptual, capitalism.

KEY WORDS:

Post internet, post-digital, neomateriality, contemporary art, conceptual art, capitalism.

\section{RESUMEN.}

El presente comunicado pretende reflexionar sobre el paradigma de una nueva neomaterialidad, condicionada por las prácticas postinternet y el escenario post-digital, y que cuestiona, de ese modo, los parámetros que marcan su propia definición. En las prácticas conceptualistas de los sesenta, consideradas el origen del linaje del arte digital, se produjo la aceptación de lo inmaterial como un objeto de estudio atado a la interacción y al proceso conceptual. Con esta alianza, lo inmaterial era tratado como un reflejo de la infinitud, como advertencia de la finitud del yo.

En el contexto post-digital se deriva hacia una continua auto-relación especular inscrita en el propio medio, en una continuidad anacrónica, donde se produce un distanciamiento de la percepción de la finitud que se tenía durante el colapso del mundo humanista. Este distanciamiento está determinado por los procesos de pluralismo formalista en los que se vieron envueltas las prácticas neoconceptualistas de los años ochenta mientras pretendían huir de la tendencia híbrida y ecléctica del arte conceptual de los años setenta.

\section{ABSTRACT.}

This statement is trying to think about the paradigm of a new neomateriality determined by the postinternet practices and the post-digital scene. By that way, this neomateriality is questioning the parametres that mark its own definition. At the conceptual artistic practices of the 60 's, considered the beggining of the digital art lineage, the immateriality was acceptated as a study object towed to the interaction and to the conceptual process. Owing to that alliance, the inmaterial was treated as a infiniteness reflect, as a warning of the self-finitness. 


\section{Porras Soriano, Álvaro y López Cleries, Gloria \\ Contradicciones del arte post-digital. Neomaterialismos vs. Infinitud \\ III CONGRESO INTERNACIONAL DE INVESTIGACIÓN EN ARTES VISUALES :: ANIAV 2017 :: GLOCAL [codificar, mediar, transformar, vivir] http://dx.doi.org/10.4995/ANIAV.2017.4895}

At the post-digital context, it derives to a continuous mirrored relation with yourself enrrolled in its own way, in an anachrinistic continuity, where the rift with the infiniteness perception that people had while the humanistic world collapse happens. This estrangement is defined by the formalist pluralist processes in which the neoconceptual practices of the 80 's were wrapped while they pretending to flee of the hybird and eclectic trend of 70 's conceptual art.

\section{CONTENIDO.}

\section{El contexto de la neomaterialidad.}

Postinternet, en su origen debate sobre un acontecimiento: la pérdida de inconexión, la cotidianeidad del Internet superficial y la operativa del networked system, lo que, en su máxima, hace deducir el salto del límite ético en la dependencia de este sistema por parte de las grandes operaciones mercantiles - transacciones, transportes y asistencia a animales humanos y no-humanos.

Observable a través de esta perspectiva, Postinternet es tratado como una época, como un momento, sincrónico con la era postdigital y posthumana. Unas condiciones de período que se subrayan bajo el momento asimilado de la postmodernidad, inscritas en la esfera del neocapitalismo conjugado entre unos estados que pierden su capacidad como nación. Esta interpretación de lo postmoderno refleja a lo posthumano como "la situación específica de las sociedades postindustriales después de la decadencia de las esperanzas y los tropos modernistas" (Braidotti, 2004: 107).

Según la lectura de Christiane Paul (2015) de las nuevas estéticas - las New Aesthetics de Bridle- que enmarcan el proceso del 'como se ve' y del 'ser visto', pero que a su vez son una serie de copias en proceso, estas producen una apariencia que cubre la putrefacción -como reflejo de finitud- del sueño modernista de la sociedad urbana civil (Braidotti, 2004: 107). Ese encubrimiento material de un anhelo nostálgico "hace referencia a un neocapitalismo sin ningún propósito teleológico, ninguna dirección definida, nada excepto la brutalidad de perpetuarse a sí mismo" (Paul, 2015).

Desde una postura posthumanista hay algo profundamente amoral y desesperado en la manera que las sociedades postindustriales se lanzan de cabeza a una solución apresurada de sus contradicciones en el momento del abandono de un mundo humanista (Braidotti, 2004: 108). Esas formas están siendo interpretadas, y también perpetuadas, por las prácticas estéticas del fenómeno Postinternet, tomadas como un conjunto de obras de arte y objetos post-digitales manifestados en formas materiales, que están conceptual y físicamente formados o ligados con respecto a Internet (Paul, 2015). Según Christiane Paul (2015), son el escaparate de una neomaterialidad que describe el pliegue de lo digital en los objetos, imágenes y estructuras a las que atendemos durante nuestra cotidianeidad, mientras que describe la manera en la que nos entendemos como individualidades y colectivos en relación con ellos.

A través de una relación basada en el comportamiento especular con un paradigma propio a cada individualidad, o con el régimen correspondiente a un periodo de vida, obtenemos una relación de temporalidad en la que el presente y nuestro yo reflejado son inmediatos, sin futuro, sin historia, solo dotados de nostalgia. Este comportamiento es, por tanto, un proceso de relación ecléctico con las concepciones de infinitud.

\section{Breve careo genealógico de la infinitud.}

Una parte notable de artistas conceptuales, dice Lippard ${ }^{1}$, tomaron como referencia a las prácticas minimalistas en cuanto a la autoproducción del cuerpo artístico, asumiendo así un nuevo enfoque industrial del emergido por el arte pop, construyéndolo limitado al cuestionamiento de la neutralidad del objeto físico en un intento de "reestructurar la percepción y la relación producto/proceso del arte" (Lippard, 2004: 17-19). En las prácticas conceptualistas de los sesenta, "a día de hoy consideradas el origen del linaje del arte digital" (Paul, 2015), se produjo la aceptación de lo inmaterial como un objeto de estudio atado a la interacción y al proceso

\footnotetext{
${ }^{1}$ La desmaterialización del arte de Lippard, a la que Christiane Paul hace referencia, contempla dos supuestos de la materia: 1) Negada, al ser la sensación convertida en concepto, y 2) Transformada por el ámbito científico en energía y espacio-tiempo. Esta revolución intermedial derivada de la desmaterialización de las prácticas de desorden conceptual tiene, según Lippard, un profeta: John Cage, quién con sus cuatro minutos y medio de silencio hacía referencia a la intrusión llevada a cabo a través de la evidenciación de un proceso marcado por la inmaterialidad, una postura adoptada también en el De Kooning borrado de Rauschenberg - en los orígenes postduchampianos del arte conceptual-, en la liberación en serie de gases inertes o la creación de campos magnéticos de Robert Barry -dentro del primer periodo conceptualista de los años sesenta-, en los rituales auto-destructivos de Meztger, Lathan, Ono y demás en el D.I.A.S -en la época en la que la objetualidad podía ser sustituida por una ausencia como una crítica política y socioeconómica-, o en el despintado de la Galería Toselli, de Michael Asher -dentro del ámbito de la crítica institucional de los años setenta. Ese trato con lo inmaterial es un reflejo procesual, en su sentido de búsqueda de lo bello natural, "de la comprensión del objeto artístico como un epílogo del concepto envolvente total"' (Lippard, 2004).
} 


\section{Porras Soriano, Álvaro y López Cleries, Gloria \\ Contradicciones del arte post-digital. Neomaterialismos vs. Infinitud \\ III CONGRESO INTERNACIONAL DE INVESTIGACIÓN EN ARTES VISUALES :: ANIAV 2017 :: GLOCAL [codificar, mediar, transformar, vivir] http://dx.doi.org/10.4995/ANIAV.2017.4895}

conceptual (Lippard y Chandler, 1968). Con esta alianza, lo inmaterial sería tratado como un reflejo de la infinitud, convocando un "estremecimiento, que está contrapuesto al concepto habitual de vivencia"(...) "y que es una advertencia de la finitud del yo" (Han, 2015: 40). Este estremecimiento sucedería, según las aspiraciones de Adorno (2005: 65), "si el arte comprendiera la ilusión de la duración y se librara de ella, si acogiera su propio carácter perecedero por simpatía con lo vivo efímero", sería entonces cuando "estaría en consonancia con una concepción de la verdad que no entiende a ésta como abstractamente permanente, sino que adquiere consciencia de su núcleo temporal".

En el contexto de lo post-digital se eliminan constantemente las negatividades de lo distinto; manteniendo un nuevo tipo de perspectiva reflejada que le obliga a que, como reverberación del contexto social que lo genera, "no deba contener ninguna rasgadura" (Adorno, 2005: 41). Esta afirmación estaría ejemplificada en los teoremas a los que harían referencia las corrientes mainstream en torno al filósofo surcoreano Byung-Chul Han tales como: el Me Gusta como máxima complacencia sin negatividad, contrapuesto por la negativa de Facebook ante el botón de No Me Gusta. Al contrario que al arte conceptual recubierto de un "aura de lejanía, que lo sustrae de todo consumo" (Paul, 2015), esa huida de la negatividad concibe que, al tratar con una producción artística inscrita en un entorno post-digital fundamentado por las re-materializaciones de las traducciones del arte Postinternet, exista un aura de cotidianeidad que hace de las relaciones neomaterialistas epistémicas una cadena de producción que las genera como materia de consumo organizada por un orden de supervivencia. Katja Novitskova, posiblemente la artista postinternet con mejor SEO en Google, sitúa a los nuevos lenguajes brotados de Internet como un algo dado, así como las carreteras o los árboles, que deben ser sentidos, interpretados e indexados para conseguir sobrevivir (Kholeif et al., 2014: 108).

Por tanto, es lógico asumir que esta materialización está condicionada por la única tolerancia hacia unas "diferencias consumibles y aprovechables" (Han, 2005: 43), que generan, en el contexto de la globalización bajo el régimen del liberalismo disciplinario, la conducción directa hacia una perenne relación especular con una auto-individualidad lineal, en una continuidad anacrónica, en la que se produce un distanciamiento de la percepción de finitud que se tenía durante el colapso del mundo humanista. Esta sensación de infinitud, de finitud esquivada, es una reforma estructurada que "enuncia un compendio de energía e información que no permiten dejar ver si, dentro sí misma, existe una propia materia que da forma a su fisicidad" (Paul, 2015), siendo esta estructura de organización social una serie de informaciones sobre las que sujetamos nuestro conocimiento y nuestra percepción extrasensorial.

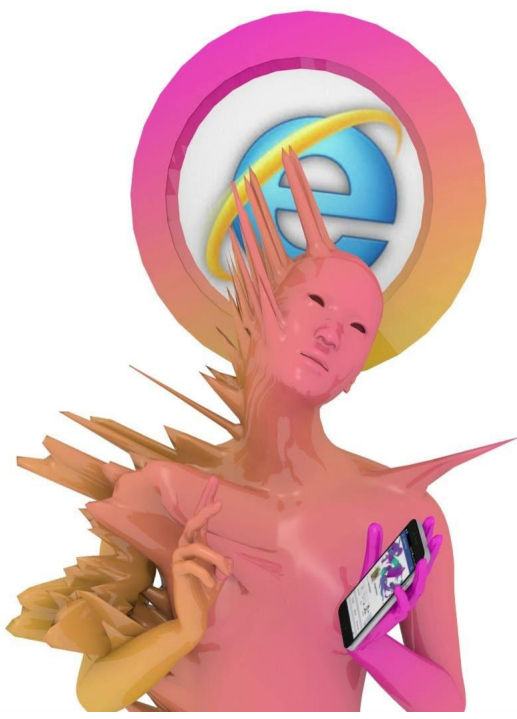

Ilustración 1. Digital Gsus, Neopantocrator, 2016. Cortesía del artista.

La situación de updating atlético que Jennifer Chan evidencia en la ansiedad existente en el arte de Internet es la respuesta que se da como una acción contraria al olvido en el contexto post-digital (Kholeif et al., 2014: 108). Esta condición conlleva, en sí, una restricción de los espacios comunes en los que es posible establecer una comunicación que no esté aislada por una comunidad. Christiane Paul (2015) reconoce en esta objetividad que incorpora la visión de nuestro mundo a través de las tecnologías en Red la descripción del concepto de neomaterialidad. Por lo tanto, el contexto neomaterialista de las prácticas artísticas que responden a Internet, se conforma mediante la formalización de unas subjetividades parciales de la comunidad, desde las que se obtiene una objetividad basada en la propia subjetivización de ese proceso formalista de las individualidades en relación con la ética neocapitalista, algo que en ojos de Adorno (2005: 33) quedaría a medio camino entre la mera estética kantiana y la epistemología del acontecimiento; en este caso, del networkhood. 


\section{Porras Soriano, Álvaro y López Cleries, Gloria \\ Contradicciones del arte post-digital. Neomaterialismos vs. Infinitud \\ III CONGRESO INTERNACIONAL DE INVESTIGACIÓN EN ARTES VISUALES :: ANIAV 2017 :: GLOCAL [codificar, mediar, transformar, vivir] http://dx.doi.org/10.4995/ANIAV.2017.4895}

A lo largo de un proceso de enfrentamiento por consecuencia entre la comunidad post-digital y las revoluciones artísticas conceptuales de los años sesenta, reconoceríamos el referente casual de las conexiones entre comunidades del periodo conceptualista en la conversación de Art Without Space guiada por el marchante Sieg Siegelaub. Ese aspecto concerniente a la comunidad podría ser adjetivado por el condicionamiento de los objetos al espacio, así como a su careo con los espacios diferentes a los espacios habituales que se experimentan al confrontar un objeto. Se propone de esta forma un cuestionamiento sobre en qué espacio de la comunidad -en dos sentidos concretos de espacio: espacio como interés específico de la obra, o espacio como condición de la conciencia de la obra- residen las producciones artísticas. Para las prácticas conceptualistas cercanas a la década de los setenta, ese espacio se encontraba en la mente de las personas que habitaban la comunidad artística cercana y habían visto la obra, y vivía mientras influenciara a más artistas (Siegelaub et al., 1969).

La portabilidad de las obras canónicas del arte conceptual que han precedido al contexto neomaterialista que presenciamos a día de hoy - referencias indudables de las recuperaciones nostálgicas neo-povera, neo-formalistas y post-conceptuales-constituyeron una apertura a la reformulación del concepto comunidad, fundamentada en la concepción efímera o inmaterial de las obras que facilitaban el viaje conjunto de la producción y quién la había generado. Lippard (2004: 23) reconoce en esa "facilidad de transporte y comunicación de las formas del arte conceptual" un hecho que favoreció el comienzo de una búsqueda de metodologías procesuales que permitieron "trabajar fuera de los principales centros artísticos y participar en los primeros foros de nuevas ideas", generando relaciones entre las práctica artísticas conceptuales del continente americano y, entre otras, las intenciones ideológicas de la Internacional Situacionista parisina, así como las de Inglaterra, Alemania o Australia antes del comienzo de la década de los setenta.

Durante la base de la globalización comercial y durante la popularización del concepto de desmaterialización conceptualista, se incorporaron a los discursos del arte las ideas volantes de relación entre validez-arte-artista, quizás descentrándose la concepción propia de las relaciones mentales como procesos de desmaterialización, quizás olvidándose que "es la inteligencia humana la que construye dichas relaciones, o, dicho de otro modo, la que crea los lenguajes" (Morgan, 2003: 54). De ese sentido estructuralista de contención, surgen las primeras prácticas de lo que Lippard y Chandler llaman arte ultra-conceptual, o arte conceptual puro: una serie de prácticas que hacen referencia a un lenguaje, o a un enfoque lingüístico, que reside "internamente dentro de la obra como medio de su arte" (Morgan, 2013: 50). Una concepción que, aunque criticada por los formalismos de final de los sesenta, ha perpetuado no por sí misma y con la sorpresa de Lippard, la desmitificación del arte hasta la eliminación de los tabús, y la inexistencia de métodos, procesos o materiales exentos para el arte, incluso hasta el punto en el que tomar el término arte conceptual funciona como un comodín (Morgan, 2013: 30) en las prácticas contemporáneas y en los discursos artísticos post-digitales. Pero, ¿podemos ejecutar un arte post-conceptual, una vez que las estructuras que fundamentaron su ruptura política han absorbido su propio proceso de reemergencia?

\section{Análisis ético en torno a la neomaterialidad.}

Aunque a los primeros grupos conceptualistas no les importara tanto el mensaje político como el divertimento filosófico, estos colectivos planteaban su práctica artística como un método de investigación paralelo a otros métodos de las ciencias sociales y naturales, pero que a su vez reformaban el proceso de crítica interna haciendo atender a los levantamientos contraculturales a los procesos de deconstrucción psicoanalítica y semiótica con la intención de generar relaciones entre la práctica artística y la idea que no podían no estar cargadas de contenido político al ser incluidas en la jerarquía de la cultura (Morgan, 2003: 32-33). El reto de las primeras prácticas conceptuales en su búsqueda de una ruptura con los sistemas formalistas, muy evidente en las obras anti-materialistas como la de Mierle Ukeles, se centraba en la interrupción de la relación entre el objeto y la mirada al negarse una relación entre arte e idea que conllevaba a la puesta en marcha de mecanismos artísticos que no parecían, en primera instancia, ni vendible ni comercial.

Esa inmaterialidad de los procesos conceptualistas forzaba a la vehicularización de los discursos artísticos como medio de composición de las relaciones entre las ideas y el contexto artístico, un proceso de desmaterialización que se producía aunque la presencia real de la obra existiera, como dice Robert Morgan (2003: 35), "hipotética o eidéticamente en forma de comunicación o enmarcando su ausencia física". Sin embargo, esta identidad del objeto artístico desmaterializado ha sido tratada, desde el resurgimiento conceptualista de los años ochenta, como una respuesta dialéctica a la lógica del capital mercantil, que involuntariamente ha acabado transformándose en un abandono de la integridad artística enunciada por Baudrillard, a favor de un marco como constructo teórico entorno a la imagen comercial. En ese proceso de construcción bourdieuana se destacan las obras que durante el proceso de globalización de la sociedad tecnológica se han "apropiado de la teoría, tanto productiva como críticamente, con la oculta presunción de que la materialización visual y conceptual de esas ideas ayude a"(...) “desenmascarar así las intenciones ocultas de la era posmoderna” (Morgan, 2003: 35).

Si bien, ¿̇puede producirse un planteamiento artístico que omita los procesos de racionalización y moralización del capitalismo? En este mismo sentido, ¿puede existir un proyecto discursivo en el que se reclame el cumplimiento del contrato moral entre el capitalismo y la sociedad que este propio sistema regula? 


\section{FUENTES REFERENCIALES.}

AdORNO, T.W. 1961-1969. Teoría Estética. En: G. ADORNO; R. TIEDEMANN (eds.) Ästhetische Theorie. 1970. Ed. Castellano: Madrid: Akal, S.A. ISBN 978-84-460-1670-0.

BRAIDOTTI, R. 2004. Feminismo, diferencia sexual y subjetividad nómade. Barcelona: Gedisa Editorial. ISBN 978-84-9784-023-1.

CHAN, J. 2013. Notes on Post-Internet. En: O. KHOLEIF (ed.). You Are Here. Art After the Internet. 2013. Manchester: Cornerhouse Publications, pp. 106-119. ISBN 978-0-9569571-7-7.

LIPPARD, L.R.; CHANDLER, J. 1968. The Dematerialization of Art. En: P. OSBORNE (ed.). Conceptual Art. 2002. New York: Phaidon Press Inc, pp.218-220. ISBN 9-780-7148-6112-8.

LIPPARD, L.R. 2004. Seis Años: La desmaterialización del objeto artístico. De 1966 a 1972. Madrid: Akal, S.A. ISBN 978-84-460-1175-0.

MORGAN, R.C. 2003. Del arte a la idea: Ensayos sobre arte conceptual. 2nd. Ed (2013). Madrid: Akal, S.A. ISBN 918-84-460-1164-4.

PAUL, C. 2015. From Immateriality to Neomateriality: Art and the Conditions of Digital Materiality. En: T. SCHIPHORST; P. PASQUIER (directors). ISEA2015: International Symposium on Electronic Art: Vancouver, Canada, August $14-19,2015$. Brighton: Inter-Society for the Electronic Arts, Proceding session no27. ISBN 978-1-910172-00-1. 